\title{
Microangiopathic haemolytic anaemia associated with recurrent pulmonary emboli and benign pelvic tumours
}

\author{
A. BLAU \\ M.B., B.S. (Melb.) \\ N. KAPLINSKY \\ M.D. \\ Department of Internal Medicine F, The Chaim Sheba Medical Center, 52621 Tel-Hashomer, Israel, \\ and The Sackler Medical School, Tel-Aviv University, Tel-Aviv, Israel
}

\begin{abstract}
Summary
A 42-year-old woman presented with a huge benign pelvic tumour and recurrent pulmonary emboli associated with severe microangiopathic haemolytic anaemia (MAHA). Following removal of the tumour and anticoagulation, all signs of MAHA disappeared. MAHA may occur when red blood cells pass through abnormal blood vessels in tumours. This patient is unusual in that the tumour was benign, rather than malignant. A further site of microangiopathy was the pulmonary vasculature.
\end{abstract}

\section{Introduction}

Microangiopathic haemolytic anaemia (MAHA) is well recognized as a rare complication of malignant tumours (Brain, Dacie and Hourihane, 1962; Antman et al., 1979). It was also described in primary pulmonary hypertension (Stuard, Heuskinkveld and Moss, 1972). We describe a woman with MAHA associated with a benign tumour, and pulmonary hypertension due to recurrent pulmonary emboli.

\section{Case history}

A 42-year-old woman was admitted in December, 1979 , because of severe dyspnoea, weakness and pain in both calves for 2 months. She was known to have a fibroid uterus for 6 years. There was no fever, cough or haemoptysis. On admission she was very pale and dyspnoeic, with a tachycardia of $124 / \mathrm{min}$, venous congestion in the neck, hepatosplenomegaly, shifting dullness in the abdomen and a huge, hard, irregular mass arising from the pelvis.

Laboratory tests revealed haemoglobin $7.8 \mathrm{~g} / \mathrm{dl}$, reticulocytes $8.5 \%$, platelet count $282 \times 10^{9} / 1$. The erythrocytes demonstrated fragmented cells, burr cells, and tear drop cells. Bone marrow biopsy revealed a hypercellular marrow with many normoblasts. Serum bilirubin $27 \cdot 2 \mu \mathrm{mol} / 1(8.5 \mu \mathrm{mol} / 1$ direct).

Liver and kidney function tests were within normal limits. Chest X-ray showed a slightly enlarged heart. The electrocardiograph was within normal limits.

Faced with a severely ill patient with a large, hard abdominal mass and severe MAHA it was assumed that the patient was suffering from a widespread malignant process.

Following blood transfusion, exploratory laparotomy was performed. A markedly enlarged uterus $\vec{C}$ with multiple necrotic masses together with bilateral ovarian tumours were removed. Histological examination revealed simple and serous cystadenoma of $\vec{\varnothing}$ the ovaries; and multiple uterine fibrotomata wi⿺辶千 cystic degeneration and necrosis. No maligna changes were seen. Postoperatively the patient's condition partially improved, but swelling of both legs was noted. At this stage with signs of phlebothrombosis, and no evidence of malignancy, an $\mathbb{D}$ explanation for the appearance of acute cor pulmunole with which the patient presented was sought. The diagnosis of recurrent pulmonary emboli was supported by the appearance of many small areas of reduced perfusion in both lung fields on perfusion lung scan. Anticoagulation with heparin was begun and continued for 2 weeks, followed by treatment with warfarin. The patient's condition improved rapidly, signs and symptoms of right heart failure regressed and there was no further evidence of $\frac{\circ}{3}$ haemolysis. One year after discharge from hospital, she is well without anticoagulation.

\section{Discussion}

Microangiopathic haemolytic anaemia was first $\tilde{N}$ described in 1962 (Brain et al., 1962), and is charac- $N$ terized by red cell fragmentation. It is observed in a $\mathrm{N}_{\mathrm{N}}$ variety of diseases associated with lesions of small $\omega$ blood vessels (Antman et al., 1979). MAHA is diffi- $\bar{Q}$ cult to treat without reversing the basic underlying $\stackrel{0}{ }$ disease process, and since it is usually associated $\stackrel{\Phi}{\mathscr{Q}}$ with diffuse malignant disease, it is frequently? irreversible. The present patient demonstrated the fact that the MAHA can be due to benign tumours, 
especially if they are large and necrotic, as has been described infrequently before (Conley, Lambird and Biesecker, 1970). MAHA is thought to occur with tumours from erythrocytes shearing on fibrin strands produced by intravascular coagulation, a mechanism that has been demonstrated experimentally as well (Hilgard and Gordon-Smith, 1974).

An alternative mechanism for the development of MAHA is shearing of erythrocytes secondary to direct contact with abnormal pulmonary vessels as has been described in the presence of pulmonary hypertension (Stuard et al., 1972). Our patient presented with dyspnoea and right heart failure as a result of recurrent pulmonary emboli. The main cause of the reversible MAHA was the huge necrotic pelvic tumour, but abnormal pulmonary vessels due to recurrent pulmonary emboli probably played a role in the pathogenesis of the MAHA.

\section{References}

Antman, K.H., Skarin, A.T., Mater, R.J. \& Hargreaves, H.K. \& CANCellos, G.R. (1979) Microangiophatic hemolytic anemia and cancer-a review. Medicine, 58, 377.

Brain, M.C., Dacie, J.V. \& Hourihane, D.O.B. (1962) Microangiopathic haemolytic anemia, the possible role of vascular lesions in pathogenesis. British Journal of Haematology, 8, 358.

Conley, C.L., Lambird, P.A. \& Biesecker, J.L.A. (1970) M.A.H.A. with recovery after removal of huge leiomyoblastoma. The John Hopkins Medical Journal, 126, 51.

Hilgard, P. \& Gordon-Smith, E. (1974) Microangiopathic haemolytic anaemia and experimental tumor emboli. British Journal of Haematology, 26, 651.

Stuard, I.D., Heuskinkveld, R.S. \& Moss, A.J. (1972) Microangiopathic hemolytic anemia and thrombocytopenia in primary pulmonary hypertension. The New England Journal of Medicine, 287, 869. 